\title{
Arthroscopic Bankart Repair with and without Arthroscopic Infraspinatus Remplissage in Anterior Shoulder Instability with Hill-Sachs Defect: Randomized Controlled Trial
}

Peter B. MacDonald, MD, FRCS ${ }^{1}$, Jason Old, MD FRCSC ${ }^{1}$, Randhir Mascarenhas, MD², Sheila McRae, PhD', Jon Marsh ${ }^{1}$, James Dubberley, MD1, Gregory A. Stranges, MD¹, Jeff Leiter, MSc, PhD1, Peter Lapner, MD³, Sharad Prabhakar, MD1

${ }^{1}$ Pan Am Clinic, Winnipeg, MB, Canada, ${ }^{2}$ University of Texas-Houston, Houston, TX, USA, ${ }^{3}$ The Ottawa Hospital, Ottawa, ON, Canada.

Objectives: The purpose of this prospective randomized, double blinded controlled trial was to compare patientreported outcomes and clinical results between arthroscopic Bankart repair with and without arthroscopic infraspinatus remplissage in patients with anterior shoulder instability with a Hill-Sachs lesion. Failure to recognize and address large Hill Sach's defects during arthroscopic stabilization surgery for glenohumeral instability is known to lead to high rates of recurrence. Arthroscopic remplissage has evolved in recent years as a reproducible technique with a proposed benefit of decreased dislocations. However, there are no high level clinical studies to conclusively support its efficacy in reducing redislocations.

Methods: One hundred and four patients, aged 14 years and older, with a confirmed Hill Sach's lesion on ultrasound, CT or MRI, were randomized intraoperatively after confirming an engaging Hill Sach's lesion to either undergo arthroscopic infraspinatus remplissage (REMP) or no remplissage during arthroscopic Bankart repair (NO REMP). Exclusion criteria included a glenoid defect $>15 \%$ of the AP glenoid diameter, significant shoulder arthropathy, infection, or medical comorbidities. The primary outcome measure was the Western Ontario Shoulder Instability score (WOSI). Secondary outcomes included the Simple Shoulder Test (SST), the American Shoulder and Elbow Society standardized assessment of shoulder function (ASES), active range of motion, stability tests, and incidence of revision surgery. Study time points were pre-, 3-, 6-, 12-, and 24-months post-operative.

Significance level was 0.05 .

Results: A summary of demographics and outcomes are presented in Table 1. There were 53 patients (45 men, 8 women) randomized to REMP and 52 (46 men, 6 women) patients to NO REMP. The groups were comparable with regard to age, body mass index (BMI), and gender distribution). Both groups demonstrated a similar improvement in all subjective scores over time to 12-months post-operative with no difference between the groups (collection of data to 24-months post-operative is ongoing until 2019). The WOSI significantly improved from pre- to 12-months post-operative in both study groups. There were no differences between groups at any time point. ASES scores and SST scores followed a similar pattern. Additionally, there were no differences in range of motion between groups at any time point. There were 2/53 re-dislocations in REMP postoperatively compared to 6/52 in NO REMP; this difference was not significant $(p=0.161)$. There were no differences between groups in reports of limitations in participation in sport attributed to the operated shoulder up to 12-months post-operative.

Conclusion: Based on this study, there is no difference in subjective outcome scores and redislocations rates between remplissage and no remplissage for an engaging Hill Sach's lesion while performing arthroscopic Bankart stabilization. As data continues to be gathered to 24 -months post-operative including MRI, longer term benefits or drawbacks may become evident.

This open-access article is published and distributed under the Creative Commons Attribution - NonCommercial - No Derivatives License (http://creativecommons.org/licenses/by-nc-nd/4.0/), which permits the noncommercial use, distribution, and reproduction of the article in any medium, provided the original author and source are credited. You may not alter, transform, or build upon this article without the permission of the Author(s). For article reuse guidelines, please visit SAGE's website at http://www.sagepub.com/journals-permissions. 
Table 1. Subjective outcomes by group - Mean (SD).

\begin{tabular}{|l|l|l|l|}
\hline & NO REMP & REMP & $p$-value \\
\hline WOSI (\%): Pre & $43(21.7)$ & $43.1(17.5)$ & 0.904 \\
\hline WOSI (\%): $12 \mathrm{mo}$ & $79.1(19.8)$ & $81.1(14.9)$ & 0.645 \\
\hline ASES (\%): Pre & $69.2(22.3)$ & $74.1(18.2)$ & 0.239 \\
\hline ASES (\%): 12 mo & $89.1(11.5)$ & $89.1(14.3)$ & 0.990 \\
\hline SST (/12): Pre & $9.1(2.4)$ & $8.9(2.8)$ & 0.675 \\
\hline SST (/12): 12 mo & $11.4(1.3)$ & $11.5(1.0)$ & 0.631 \\
\hline Limited by shoulder in return to sport (0-100): 12 mo & $27.4(31.3)$ & $20.9(24.3)$ & 0.351 \\
\hline
\end{tabular}

The Orthopaedic Journal of Sports Medicine, 6(7)(suppl 4)

DOI: $10.1177 / 2325967118 S 00095$

(C)The Author(s) 2018 